\title{
Hypergravity affects Cell Traction Forces of Fibroblasts
}

\author{
J. Eckert ${ }^{1,2, *}$, J. J.W.A. van Loon ${ }^{3,4}$, L. M. Eng ${ }^{2}$, and T. Schmidt ${ }^{1}$
}

\begin{abstract}
Cells sense and react on changes of the mechanical properties of their environment, and likewise respond to external mechanical stress applied to them. Whether the gravitational field, as overall body force, modulates cellular behavior is however unclear. Different studies demonstrated that micro- and hypergravity influences the shape and elasticity of cells, initiate cytoskeleton reorganization, and influence cell motility. All these cellular properties are interconnected, and contribute to forces that cells apply on their surrounding microenvironment. Yet, studies that investigated changes of cell traction forces under hypergravity conditions are scarce. Here we performed hypergravity experiments on 3T3 fibroblast cells using the Large Diameter Centrifuge at the European Space and Technology Centre (ESA-ESTEC). cells were exposed to hypergravity of up to $19.5 \mathrm{~g}$ for $16 \mathrm{~h}$ in both the upright and the inverted orientation with respect to the g-force vector. We observed a decrease in cellular traction forces when the gravitational field was increased up to $5.4 \mathrm{~g}$, followed by an increase of traction forces for higher gravity fields up to $19.5 \mathrm{~g}$ independent of the orientation of the gravity vector. We attribute the switch in cellular response to shear-thinning at low $\mathrm{g}$-forces, followed by significant rearrangement and enforcement of the cytoskeleton at high g-forces.
\end{abstract}

SIGNIFICANCE The behavior of cells critically depend on the mechanical properties of their environment. For example external stresses and strains lead to decisions in cell differentiation as well as to collective-migration in metastasis. Gravity, as a permanently acting body force, is one of those externs stresses. We demonstrate the impact of gravitational challenges on forces that cells apply to their environment. We observed a switch in cellular response with a decrease in cell traction forces for low bypgrarayiv. conditions, followed by a significant increase in cell traction forces at higher g-level. This particular cellular response reflects a switch in croskeletal organization, similar to that observed for cells in fluids where shear forces act.

\section{INTRODUCTION}

In recent years it became accepted that cellular function is, in part, controlled by external mechanical cues. Mechanical cues were shown to be sufficient to differentiate mesenchymal stem cells (1), initiate transcriptional programs (2), drive morphogenesis (3), direct cell migration (4), and control malignancy in tumors (5). The force and stiffness-mediated responses of cells on the mechanical properties of the extracellular matrix (ECM) are attributed to, yet to be identified, mechano-chemical sensor platforms that transform external mechanical cues into intracellular biochemical signals, ultimately leading to e.g. altered gene expression (6). The multi-protein sensory units responsible for mechano-sensation are summarized as cell-matrix adhesions, focal adhesions, and cell-cell adhesions (7). In focal adhesions, transmembrane receptor proteins such as integrins (8), bind to specific proteins of the ECM. On the cytosolic side those proteins link, through a extended protein-cascade, to the actin cytoskeleton, the cellular machinery that can apply forces through the contraction of actin fibers through the linking myosin motor-activity (9-11).

So far, studies on the mechano-chemical coupling have focused on cellular responses related to static extracellular stiffness (12) and topography (13), as well as on the direct mechanical stimulation of cells by fluid flow (14), micropipette aspiration (15) (16), optical tweezers (17), optical stretchers (18), atomic-force microscopes (19), and magnetically actuated particles (20). In most of those experiments local stress was applied to the cells that resulted in a highly sensitive cellular response. Cells were shown to adapt to their local mechanical environment by enforcing their cytoskeleton. Such restructuring of the cytoskeleton 
is paralleled by an increased force application of cells when placed into stiffer environments or when challenged by higher external tensions.

Surprisingly, the robust cellular response on localized mechanical cues appears more subtle for homogeneous mechanical cues such as that given by gravity. Experiments showed that hypergravity in the range of 2-20g does influence cellular morphology and elasticity (21) (22), the cytoskeletal organization (23) and the motility (24). The strength and impact, however, differ largely, as was reviewed for endothelial cells by Maier et al. (25). The effects observed depend on many factors, which appeared difficult to disentangle. Next to different cell types, different g-levels applied, and different exposure times of challenge, in particular, the use of small laboratory centrifuges by which hypergravity was produced are known to generate additional mechanical cues on cells (in particular internal shear-forces) that possibly overshadow the effects of gravitational cues (26).

Monici et al. exposed microvascular endothelial cells for a period of $5 \times 10 \mathrm{~min}$ to $10 \mathrm{~g}$. Cells were centrifuged under closed conditions in a thermostated laboratory centrifuge. In the experiment, the authors showed changes in the cytoskeleton organization (23). The opposite was reported by Costa-Almeida et al (27). In the latter experiment endothelial cells (HUVEC) were exposed exposed to 3 and $10 \mathrm{~g}$ for 4 and $16 \mathrm{~h}$ using the large-diameter centrifuge (LDC) at the ESA/ESTEC center in Noordwijk. No changes in cytoskeleton organization was observed.

Here, we focus on investigating the impact of hypergravity as a body force on the active cellular mechano-response. Given that hypergravity has an influence on the cell morphology (21) (22), cytoskeleton organization (23), membrane viscosity (28), and motility (24), we probed whether or not cells react on hypergravity by a modulation of their traction forces towards the ECM.

\section{MATERIALS AND METHODS}

\section{Cell Culture}

3T3 fibroblasts were cultured in high-glucose Dulbecco's Modified Eagle's Medium (DMEM, Sigma Aldrich) supplemented with $10 \%$ fetal calf serum (FCS, Thermo Fisher Scientific), $2 \mathrm{mM}$ glutamine and $100 \mu \mathrm{g} / \mathrm{ml}$ penicillin/streptomycin, $37^{\circ} \mathrm{C}$, $5 \% \mathrm{CO}_{2}$.

\section{Immunostaining}

$5 \mathrm{~min}$ after the hypergravity exposure, cells were fixed for $15 \mathrm{~min}$ in $4 \%$ paraformaldehyde (Alfa Aesar, 43368) in phosphatebuffered saline (PBS). After fixation, cells were permeabilized for $10 \mathrm{~min}$ with $0.1 \%$ Triton-X and blocked for 60 min with $1 \%$ BSA in PBS. F-actin was stained with Alexa Fluor 532-labelled phalloidin (Invitrogen, A22282) and the DNA with DAPI (Sigma).

\section{Hypergravity Exposure}

In order to avoid shear forces (26), hypergravity experiments were performed using the Large Diameter Centrifuge (LDC, Fig. 1A) at the European Space Research and Technology Center in Noordwijk, The Netherlands. Cells were seeded on two sets of elastic micropillar arrays, located in 12-well-plates with one array per well, and incubated for $5.5 \mathrm{~h}, 37^{\circ} \mathrm{C}, 5 \% \mathrm{CO}_{2}$. After cell spreading on top of the functionalized micropillars, arrays of one set were flipped in the up-side-down orientation. Both sets were placed in closed metal boxes flushed with $5 \% \mathrm{CO}_{2}$ at $100 \%$ humidity and stored inside the incubators of the LDC-gondolas held at $35-37^{\circ} \mathrm{C}$ (Fig. 1B). The centrifuge gondolas were placed at a distance of 2 and $4 \mathrm{~m}$ to the centrifuge axis, which allowed us to address two g-levels, simultaneously (29). $6.5 \mathrm{~h}$ after cell seeding, they were exposed for $16 \mathrm{~h}$ to hypergravity of $5.4 \mathrm{~g}, 10 \mathrm{~g}$ and $19.5 \mathrm{~g}$, respectively, as controlled by the distance of the gondolas from the axis, and the speed at which the centrifuge turned. As of the large-diameter of the centrifuge, shear-forces were negligible in our experiments (26). The g-force acted perpendicular to the sample surface (Fig. 1C). $1 \mathrm{~g}$ control experiments were prepared and conducted simultaneously under identical conditions outside the centrifuge.

\section{Elastic Micropillar Arrays}

Polydimethylsiloxane (PDMS, Sylgard 184) micropillar arrays of $2 \mu \mathrm{m}$ diameter, $6.9 \mu \mathrm{m}$ height and $4 \mu \mathrm{m}$ spacing in a hexagonal geometry were used for cell traction force experiments. The pillar arrays were flanked by $50 \mu \mathrm{m}$ spacers on two sides of the array. Details of this arrangement and the experimental procedures was described earlier in detail (30). In brief, pillar arrays were produced on a negative silicon-wafer master made by a two-step deep reactive-ion etching process. Wafers were passivated in trichloro-silane (Sigma Aldrich, 448931). A mixture of 1:10 PDMS (crosslinker:base ratio) was poured onto the Si-master and cured for $20 \mathrm{~h}$ at $110^{\circ} \mathrm{C}$. After peel-off, the tops of the pillars were coated by $\mu$-contact printing. For that, flat 1:30 PDMS 
stamps were incubated for $1 \mathrm{~h}$ with $40 \mu \mathrm{l}$ of $50 \mu \mathrm{g} / \mathrm{ml}$ Alexa Fluor 647 labelled and $50 \mu \mathrm{g} / \mathrm{ml}$ unlabelled fibronectin (Sigma Aldrich, F1141), then washed and dried. Subsequently, the stamps were gently loaded onto the UV-ozone-activated micropillar arrays for $10 \mathrm{~min}$. After stamping, the arrays were passivated with $0.2 \%$ Pluronic (F-127, Sigma Aldrich, P2443) for $1 \mathrm{~h}$, and washed in phosphate-buffered saline (PBS).

\section{Microscopy}

Samples were imaged at high resolution on a home-build optical microscope setup based on an inverted Axiovert200 microscope body (Zeiss), a spinning disk unit (CSU-X1, Yokogawa), and an emCCD camera (iXon 897, Andor). IQ-software (Andor) was used for setup-control and data acquisition. Illumination was performed using fiber-coupling of different lasers $(405 \mathrm{~nm}$ (CrystalLaser), $514 \mathrm{~nm}$ (Cobold) and $642 \mathrm{~nm}$ (Spectra-Physics Excelsior)). Pillar arrays were placed up-side-down onto $25 \mathrm{~mm}$ cover glasses and inspected with an EC Plan-NEOFLUAR 40x1.3 Oil immersion objective (Zeiss).

\section{Image Analysis}

Images were analysed using Matlab scripts (Mathworks, Matlab R2017a). Pillar deflections were quantified as previously described in detail (30). The accuracy of the analysis was determined from an undeflected area of the pillar array by selecting a pillar region outside the cell area. Pillar deflections underneath the cell within the background range were discarded. The traction force per pillar was calculated by dividing the total absolute force per cell by the number of deflected pillars per cell. The cell spreading area was calculated as the number of deflected pillars per cell multiplied by the unit-cell area of the hexagonal pillar array geometry. The unit-cell area measured $13.84 \mu \mathrm{m}^{2}$.

Additionally, we calculated the bending modulus of pillars caused by the increase in the weight of the cell and the pillar itself at higher g-level (see supplemental material). Based on the study by Grover $e t$ al., we assumed a cell density of $1.08 \mathrm{~g} \mathrm{ml}^{-1}$ (31). The averaged diameter of $3 \mathrm{~T} 3$ fibroblasts is $50 \mu \mathrm{m}$ with a height of $15 \mu \mathrm{m}$ measured via z-stack images. Assuming a deflection of $400 \mathrm{~nm}$, the differences of the pillar deflection at $1 g$ to that at $20 \mathrm{~g}$ is $4.7 \mathrm{pN}$ (S5). Hence, the additional pillar deflection is 3 orders of magnetude lower than that resulting from cellular traction force, and can thus be neglected.

\section{Statistics}

The following samples with cells were analyzed in the upright orientation: Two control arrays with 101 cells at $1 g$, one array with 72 cells at $5.4 g$, two arrays with 112 cells including one repeat at $10 g$, and one array with 66 cells at $19.5 g$.

In the up-side-down orientation, we analyzed: Two control arrays with 100 cells at $1 g$, one array with 65 cells at $5.4 g$, one array with 54 cells at $10 \mathrm{~g}$, and one array with 47 cells at $19.5 \mathrm{~g}$. Hence, in total 617 cells and 20343 deflections were analyzed.

P-values were calculated using the two-sided Wilcoxon rank sum test in Matlab. Data sets were significantly different with probabilities of $\mathrm{p} \leq 0.05(*) ; \mathrm{p}<0.01(* *) ; \mathrm{p}<0.001(* * *)$.

\section{RESULTS}

Given the results of prior studies on the effect of hypergravity on cellular behavior (21) (23) (24), we anticipated that the effect of hypergravity on cellular force application would be small. Hence, we initially performed an extensive analysis of cell traction forces using the micropillar technique to extract a solid, experimentally confirmed base-line value for all parameters investigated.

Cells were seeded on six independently produced arrays and left for $6 \mathrm{~h}$. After fixation and staining for actin and DNA, 323 cells leading to 9545 pillar deflections were analyzed. In a first step, the total absolute force that a cell produces was compared to the number of deflected pillars for that particular cell. The data are shown in Fig. 2A. The total force per cell appeared highly correlated to the number of deflected pillars $(|r|>0.8)$. From this correlation, we concluded that the mean force per pillar is a robust descriptor for cellular force application. This conclusion corroborates earlier experiments in which it was shown that the force per pillar is a cellular property which depends on external chemical or mechanical cues (32). Hence, in what follows, we calculated the mean traction forces per deflected pillar.

In how far the mean traction force per pillar is a robust quantity with respect to the production of micropillar arrays, we compared data from six independently produced arrays (Fig. 2B). Mean values for the force per pillar varied for each array between $(10.4 \pm 3.4) \mathrm{nN}$ (array 1, 59 cells) and $(15.1 \pm 7.8) \mathrm{nN}$ (array 4, 39 cells). A two-sided Wilcoxon rank sum test confirmed that the data set of each of the array was different. Hence, this result suggests that biological and technological (e.g. pillar geometry) variability in our data could easily be underestimated. In what follows, we thus defined results of cellular forces that fall into the range $10.4-15.1 \mathrm{nN}$ as indistinguishable from the control experiment at $1 g$-condition.

Further, we compared the cell size to the cellular force per pillar (Fig. 2C). The cell size was calculated from the cell 
spreading area as seen in the actin-channel of the images taken. In contrast to the strong correlation between total cell force and number of deflected pillars in Fig. 2A, the correlation coefficient between force per pillar and cell size was small, $0.05<|r| \leq 0.27$. Hence, taken both results into account we concluded, that the mean force per deflected pillar is a robust measure of cellular forces when technological variability is properly considered.

Subsequently, we compared the $1 g$ results to those obtained for hypergravity conditions. Cell-loaded micropillar arrays immersed in 12-well-plates were placed into the incubators of two gondolas of the large diameter centrifuge (LDC) at ESA/ESTEC. Together with the control at standard $1 g$ gravity condition, we exposed cells to three different g-levels. For statistical reasons, we performed two independent experiments for $16 \mathrm{~h}$ each at $1 g, 5.4 \mathrm{~g}$ and $10 \mathrm{~g}$ and one experiment at $1 g, 10 \mathrm{~g}$ and $19.5 \mathrm{~g}$, respectively. The g-vector acted perpendicular to the cell spreading area. Arrays were located in both the upright (positive g-vector) and the up-side-down (negative g-vector) orientation. For analysis, cells were fixed, stained and imaged remotely.

As predicted from our $1 \mathrm{~g}$ experiments, the total force per cell highly correlated with the number of deflected pillars also for hypergravity conditions for both the upright and the upside-down orientation. Data for the upright orientation (positive g-vector) are shown in Fig. 3A. The correlation coefficient of $0.8 \leq|r| \leq 1$ was equivalent to that we found for $1 g$. In addition, we verified that the force per pillar was independent on the cell spreading area. The correlation is shown in Fig. 3D for two sets at two different g-levels. The data were uncorrelated as inferred from the low value of the correlation coefficient, $|r|<0.11$. Hence, as for the $1 \mathrm{~g}$ condition, the mean force per deflected pillar is a robust measure to characterize cellular forces also for hypergravity conditions.

In turn, we analyzed the averaged force per deflected pillar for the various hypergravity levels (Fig. 3B-C). In both orientations, the force per pillar decreased significantly when hypergravity was changed from $1 \mathrm{~g}$ to $5.4 \mathrm{~g}$. When hypergravity was increased further to $10 \mathrm{~g}$ and $19.5 \mathrm{~g}$, respectively, the force per pillar increased again, finally exceeding the value at $1 g$. In the upright orientation (positive g-vector, Fig. 3B), cells applied significantly less force on pillars at 5.4g with an average of $(8.1 \pm 2.0) \mathrm{nN}$ and $(9.1 \pm 3.7) \mathrm{nN}$ at $10 g$, respectively. At $19.5 g$, the traction force per deflected pillar with an average of $(12.9 \pm 5.4) \mathrm{nN}$ was not significantly different from $1 g$ with $(12.7 \pm 4.8) \mathrm{nN}$. The strongest effect and biggest difference was measured for cells in up-side-down orientation (negative g-vector, Fig. 3C). In this orientation, cells applied the lowest force per pillar at $5.4 g$ with an average of $(8.3 \pm 3.5) \mathrm{nN}$ and the highest force with $(15.3 \pm 5.0) \mathrm{nN}$ at $19.5 g$, which is visualized in Fig. 3E-F. The traction force of the $1 g$ control with $(13.3 \pm 4.9) \mathrm{nN}$ was significantly different to both results at $5.4 g$ and $19.5 g$, while it was equivalent to the averaged force of $(12.3 \pm 4.4) \mathrm{nN}$ at $10 \mathrm{~g}$. A two-sided Wilcoxon rank sum test was used to compare the two orientations shown in Fig. 3B-C. The force per pillar did not differ significantly for the $1 g$ and the 5.4 $g$ situations. Yet, at higher g-levels the two configurations were significantly different, with $p<0.0001$ at $10 g$, and $p=0.0044$ for $19.5 g$.

A clearer picture of the significance of the different results was obtained from the analysis of the cumulative distribution function of the mean force per pillar values. From the six $1 g$-control measurements, we constructed a significance-band for the cumulative distribution (gray area in Fig. 3G). The band was constructed such that all six $1 g$-control measurements fall into the given range. The forces of cells in the upright orientation exposed to $5.4 \mathrm{~g}$ and $10 \mathrm{~g}$ are fully outside of the gray range, while $1 \mathrm{~g}$ and $19.5 \mathrm{~g}$ samples are equal to the standard gravity distributions. This confirms our results of the change of cell traction forces under hypergravity conditions when analyzed by a Wilcoxon test (Fig. 3B).

\section{DISCUSSION}

It has been shown that hypergravity influences cells morphology and behavior. Although the outcome may highly vary (25) it was well documented that hypergravity leads to a rearrangement of the cytoskeleton (23)(27). Here, we investigated in how far hypergravity modulates the contractile behavior of cells. Given that the cytoskeleton represents the main contractile machinery within cells, we anticipated that forces exerted by cells onto their environment, would likewise change.

We cultured 3T3 fibroblasts on elastic micropillar arrays and exposed them for $16 \mathrm{~h}$ to hypergravity at a range of g-levels. As we predicted, cellular forces changed for hypergravity conditions: we found an initial decrease of forces from $1 \mathrm{~g}$ to $5.4 \mathrm{~g}$, followed by a subsequent increase in forces at least up to $19.5 \mathrm{~g}$.

Our results corroborate earlier findings elucidating the reorganization of the actin network. Versari et al. cultured epithelial cells (HUVEC) in a medium-sized centrifuge for acceleration research (MidiCAR) and found less dense actin fibers after $96 \mathrm{~h}$ at $3.5 \mathrm{~g}(33)$. The decrease in actin stress fibers at low hypergravity levels confers with our results on a decrease in traction forces at 5.4g. Also the more recent results by Costa-Almeida et al, who exposed human tendon-derived cells (hTDCs) to $5 g$,

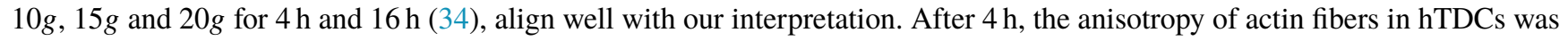
significantly lower at $5 g$ as compared to $1 g$ and increased towards higher g-levels.

Other external stress measurements showed a similar effect on the actin filament formation. Kataoka et al. performed flow-imposed experiments. Using a parallel flow plate chamber, endothelial cells were exposed to different flow directions for $24 \mathrm{~h}$. Under fluid shear stress as low as $2 \mathrm{~Pa}$, cells perfectly aligned with the flow direction and formed thick stress fibers (35). 
Kuo et al. applied an oscillatory shear stress with a frequency of $1 \mathrm{~Hz}$ on cells. Under lower shear stress, the phalloidin-labelled F-actin signal decreases at $0.05 \mathrm{~Pa}(36)$ after $0.5 \mathrm{~h}$, which indicates a reorganization of actin filaments. In terms of the cell volume (density: $1.08 \mathrm{~g} \mathrm{ml}^{-1}$ (31), height: $15 \mu \mathrm{m}$ ), $1 \mathrm{~g}$ can be assigned to a shear stress of $0.16 \mathrm{~Pa}, 10 \mathrm{~g}$ to $1.6 \mathrm{~Pa}$ and $20 \mathrm{~g}$ to 3.2 Pa. Hence, fluid-shear stress and hypergravity seem to have a similar impact on the actin reorganization of cells and possibly on cell traction forces. On the other hand, Perrault et al. measured an increase in strain energy independent from the flow rate $(0.014-0.133 \mathrm{~Pa})(37)$. Care must be taken that fluid-shear stress as a surface force acts parallel to the spread cell and only on the affected surface area. In contrast, hypergravity as a body force acts perpendicular to it and on all parts of the cell volume.

Further it was found that the internal organization of the cytoskeleton alters with mechanical challenge. Norstrom et al. studied shear thickening of cross-linked F-actin networks. Performing rheological experiments, the authors observed viscous deformation of stresses from 0.001 to $10 \mathrm{~Pa}$, caused by stress stiffening and shear thickening. To a surprise, and in contrast to earlier findings in which stress weakening of sparsely cross-linked actin network was measured, Norstrom et al. observed a stress stiffening behaviour of a densely cross-linked network (38). This finding is consistent with the work of Gardel et al. (39) who highlighted the connection between the elasticity of the actin network, the density of cross-linkers and the actin concentration.

Combining the data of Norstrom et al. and Gardel $e$ al. with our findings, an interaction between the external g-level acting on the cell and the elasticity of it's actin network appears apparent. At low hypergravity levels, a less dense cross-linked actin network with less oriented stress fibers might be formed. This would result in a decrease in cell traction forces. At larger g-levels, the actin network would then form oriented densely-packed stress fibers, which are highly cross-linked. Those densely-packed, highly cross-linked stress fibers will result in higher traction forces due to stress stiffening.

Hence, based on our results, we propose that hypergravity causes a re-organization of the actin network dependent on the g-level, where gravitation acts similar to that reported for fluid-shear stress. Reorganization of the cytoskeleton subsequently causes a change in traction force that was observed in our experiments.

\section{CONCLUSION}

In conclusion we demonstrated that hypergravity modulates the traction force of 3T3 fibroblasts. Dependent on the g-force level, the cell traction force first decreases for low hypergravity conditions, yet increases for higher g-levels. We found that cells in up-side-down orientation were more affected when compared to cells in upright orientation. We propose that the change in cellular force-response reflects the reorganization of the cytoskeleton as triggered by a gravitational cue, very similar to cellular responses to fluid shear-flow. Further studies should be employed to investigate the involvement of e.g. the myosin activity, and the actin stress fiber formation on the force transduction at altered gravity conditions. Our data should be considered in order to estimate potential health-riscs in planned long-haul space flights.

\section{AUTHOR CONTRIBUTIONS}

sample preparation: JE

conducted hypergravity experiments: JE, JJWAL

data analysis: JE, TS

writing manuscript: JE, JJWAL, LME, TS

initiated the study: JJWAL

All authors gave final approval for publication.

\section{ACKNOWLEDGMENTS}

We acknowledge Robert Lindner and Alan Dowson (TEC-MMG, ESA/ESTEC) for the support and access to the LIS-Lab and the LDC. J.E. thanks for the support of an Erasmus+ fellowship.

\section{REFERENCES}

1. Engler, A. J., S. Sen, H. L. Sweeney, and D. E. Discher, 2006. Matrix Elasticity Directs Stem Cell Lineage Specification. Cell 126:677-689.

2. Farge, E., 2003. Mechanical Induction of Twist in the Drosophila Foregut/Stomodeal Primordium. Current Biology 13:1365-1377.

3. Bosveld, F., I. Bonnet, B. Guirao, S. Tlili, Z. Wang, A. Petitalot, R. Marchand, P.-L. Bardet, P. Marcq, F. Graner, and Y. Bellaïche, 2012. Mechanical Control of Morphogenesis by Fat/Dachsous/Four-Jointed Planar Cell Polarity Pathway. Science 336:724-727. 
Eckert et al.

4. Sunyer, R., V. Conte, J. Escribano, A. Elosegui-Artola, A. Labernadie, L. Valon, D. Navajas, J. M. García-Aznar, J. J. Muñoz, P. Roca-Cusachs, and X. Trepat, 2016. Collective cell durotaxis emerges from long-range intercellular force transmission. Science 353:1157-1161.

5. Miroshnikova, Y. A., J. K. Mouw, J. M. Barnes, M. W. Pickup, J. N. Lakins, Y. Kim, K. Lobo, A. I. Persson, G. F. Reis, T. R. McKnight, E. C. Holland, J. J. Phillips, and V. M. Weaver1, 2016. Tissue mechanics promote IDH1-dependent HIF1 $\alpha$-tenascin C feedback to regulate glioblastoma aggression. Nature cell biology 18:1336-1345.

6. Balaban, N. Q., U. S. Schwarz, D. Riveline, P. Goichberg, G. Tzur, I. Sabanay, D. Mahalu, S. Safran, A. Bershadsky, L. Addadi, and B. Geiger, 2001. Force and focal adhesion assembly: a close relationship studied using elastic micropatterned substrates. Nature Cell Biology 3:466-472.

7. Yamada, K. M., and B. Geiger, 1997. Molecular interactions in cell adhesion complexes. Current Opinion in Cell Biology 9:76-85.

8. Hynes, R. ., 1992. Integrins: versatility, modulation, and signaling in cell adhesion. Cell 69:11-25.

9. Burridge, K., and M. Chrzanowska-Wodnicka, 1996. Focal Adhesion, Contractility, and Signaling. Annual Review of Cell and Developmental Biology 12:463-519.

10. Yamada, K. M., and S. Miyamoto, 1995. Integrin transmembrane signaling and cytoskeletal control. Curr. Opin. Cell 7:681-689.

11. Veigel, C., and C. F. Schmidt, 2011. Moving into the cell: single-molecule studies of molecular motors in complex environments. Nature Reviews Molecular Cell Biology 12:163-176.

12. Georges, P. C., and P. A. Janmey, 2005. Cell type-specific response to growth on soft materials. Journal of Applied Physiology 98:1547-1553.

13. Lehnert, D., B. Wehrle-Haller, C. David, U. Weiland, C. Ballestrem, B. A. Imhof, and M. Bastmeyer, 2004. Cell behaviour on micropatterned substrata: limits of extracellular matrix geometry for spreading and adhesion. Journal of Cell Science $117: 41-52$.

14. Paddillaya, N., A. Mishra, P. Kondaiah, P. Pullarkat, G. I. Menon, and N. Gundiah, 2019. Biophysics of Cell-Substrate Interactions Under Shear. Frontiers in cell and developmental biology 7:251.

15. Evans, E. A., 1973. New membrane concept applied to the analysis of fluid shear- and micropipette-deformed red blood cells. Biophysical journal 13:941-954.

16. Hochmuth, R. M., N. Mohandas, and P. L. Blackshear, 1973. Measurement of the elastic modulus for red cell membrane using a fluid mechanical technique. Biophysical journal 13:747-762.

17. Ashkin, A., J. M. Dziedzic, and T. Yamane, 1987. Optical trapping and manipulation of single cells using infrared laser beams. Nature 330:769-771.

18. Guck, J., R. Ananthakrishnan, H. Mahmood, T. J. Moon, C. C. Cunningham, and J. Käs, 2001. The optical stretcher: a novel laser tool to micromanipulate cells. Biophysical journal 81:767-784.

19. Kasas, S., P. Stupar, and G. Dietler, 2018. AFM contribution to unveil pro- and eukaryotic cell mechanical properties. Seminars in Cell \& Developmental Biology 73:177-187.

20. Hu, S., L. Eberhard, J. Chen, J. C. Love, J. P. Butler, J. J. Fredberg, G. M. Whitesides, and N. Wang, 2004. Mechanical anisotropy of adherent cells probed by a three-dimensional magnetic twisting device. American Journal of Physiology-Cell Physiology 287:C1184-C1191.

21. Tavakolinejad, A., M. Rabbani, and M. Janmaleki, 2015. Effects of hypergravity on adipose-derived stem cell morphology, mechanical property and proliferation. Biochemical and Biophysical Research Communications 464:473 - 479.

22. van Loon, J., M. van Laar, J. Korterik, F. Segerink, R. Wubbels, H. de Jong, and N. van Hulst, 2009. An atomic force microscope operating at hypergravity for in situ measurement of cellular mechano-response. Journal of microscopy 233:234-243. 
23. Monici, M., N. Marziliano, V. Basile, G. Romano, A. Conti, S. Pezzatini, and L. Morbidelli, 2006. Hypergravity affects morphology and function in microvascular endothelial cells. Microgravity - Science and Technology 18:234-238.

24. Croute, F., Y. Gaubin, B. Pianezzi, and J. P. Soleilhavoup, 1994. Effects of hypergravity on the morphology, the cytoskeleton, the synthesis of extracellular macromolecules and the activity of degradative enzymes. Life Sciences Research in Space, Proceedings of the Fifth European Symposium held 26 September - 1 October, 1993 in Arcachon, France. Edited by H. Oser and T.D. Guyenne. ESA SP-366. European Space Agency 31-34.

25. Maier, J. A. M., F. Cialdai, M. Monici, and L. Morbidelli, 2015. The Impact of Microgravity and Hypergravity on Endothelial Cells. BioMed Research International 2015:13 pages.

26. van Loon, J. J. W. A., E. H. T. E. Folgering, C. V. C. Bouten, T. H. Smit, and T. H. Smit, 2003. Inertial Shear Forces and the Use of Centrifuges in Gravity Research. What is the Proper Control? Journal of Biomechanical Engineering 125:342-346.

27. Costa-Almeida, R., D. T. O. Carvalho, M. J. S. Ferreira, G. Aresta, M. E. Gomes, J. J. W. A. van Loon, K. Van der Heiden, and P. L. Granja, 2016. Effects of hypergravity on the angiogenic potential of endothelial cells. Journal of The Royal Society Interface 13.

28. Woodcock, E. M., P. Girvan, J. Eckert, I. Lopez-Duarte, M. Kubánková, J. J. van Loon, N. J. Brooks, and M. K. Kuimova, 2019. Measuring Intracellular Viscosity in Conditions of Hypergravity. Biophysical Journal 116:1984-1993.

29. van Loon, J. J., and A. Dowson, 2019. Large Diameter Centrifuge (LDC) Experimenter Users Manual. ESA-TECMMGMAN-014129 3:1-33.

30. van Hoorn, H., R. Harkes, E. M. Spiesz, C. Storm, D. van Noort, B. Ladoux, and T. Schmidt, 2014. The Nanoscale Architecture of Force-Bearing Focal Adhesions. Nano Letters 14:4257-4262.

31. Grover, W. H., A. K. Bryan, M. Diez-Silva, S. Suresh, J. M. Higgins, and S. R. Manalis, 2011. Measuring single-cell density. Proceedings of the National Academy of Sciences 108:10992-10996.

32. Balcioglu, H. E., H. van Hoorn, D. M. Donato, T. Schmidt, and E. H. J. Danen, 2015. The integrin expression profile modulates orientation and dynamics of force transmission at cell-matrix adhesions. Journal of Cell Science 128:1316-1326.

33. Versari, S., A. Villa, S. Bradamante, and J. A. Maier, 2007. Alterations of the actin cytoskeleton and increased nitric oxide synthesis are common features in human primary endothelial cell response to changes in gravity. Biochimica et Biophysica Acta (BBA) - Molecular Cell Research 1773:1645-1652.

34. Costa-Almeida, R., D. T. Carvalho, M. J. Ferreira, T. Pesqueira, M. Monici, J. J. van Loon, P. L. Granja, and M. E. Gomes, 2018. Continuous Exposure to Simulated Hypergravity-Induced Changes in Proliferation, Morphology, and Gene Expression of Human Tendon Cells. Stem Cells and Development 27:858-869.

35. Kataoka, N., S. Ujita, and M. Sato, 1998. Effect of flow direction on the morphological responses of cultured bovine aortic endothelial cells. Medical and Biological Engineering and Computing 36:122-128.

36. Kuo, Y.-C., T.-H. Chang, W.-T. Hsu, J. Zhou, H.-H. Lee, J. Hui-Chun Ho, S. Chien, and O. Kuang-Sheng, 2015. Oscillatory Shear Stress Mediates Directional Reorganization of Actin Cytoskeleton and Alters Differentiation Propensity of Mesenchymal Stem Cells. STEM CELLS 33:429-442.

37. Perrault, C. M., A. Brugues, E. Bazellieres, P. Ricco, D. Lacroix, and X. Trepat, 2015. Traction Forces of Endothelial Cells under Slow Shear Flow. Biophysical Journal 109:1533-1536.

38. Norstrom, M., and M. L. Gardel, 2011. Shear thickening of F-actin networks crosslinked with non-muscle myosin IIB. Soft Matter 2011:3228-3233.

39. Gardel, M. L., J. H. Shin, F. C. MacKintosh, L. Mahadevan, P. Matsudaira, and D. A. Weitz, 2004. Elastic Behavior of Cross-Linked and Bundled Actin Networks. Science 304:1301-1305. 
Eckert et al.

\section{SUPPLEMENTARY MATERIAL \\ Beam Theory under Hypergravity Conditions}

The deflection of the micropillar as an elastostatic process is described by the Bernoulli beam theory. Perpendicular acting forces to the beam axis with small angular changes are described by the Euler-Bernoulli assumption for narrow beams. The deflection of the beam $\delta:=\omega(x)$ is calculated from the curvature of the bending line as a differential equation:

$$
\omega^{\prime \prime}(x)=-\frac{M}{E I} \text {. }
$$

$M$ is the bending moment, $E$ the Young's modulus and $I$ the second momentum of area:

$$
I=\frac{\pi}{64} d^{4}
$$

including the beam diameter, $\mathrm{d}$. Taking the boundary conditions for a beam with clamping $\omega(0)=0$ and $\omega^{\prime}(0)=0$, the lateral force acting on the pillar is described by

$$
F=\frac{3 E I}{h^{3}} \delta
$$

with the pillar height.

Gravity causes additional forces acting on the beam (Fig. 4 A): the gravitational force of the pillar, $F_{\mathrm{g}, \mathrm{Pillar}}$, and of the cell, $F_{\mathrm{g}, \text { Cell }}$, both are calculated by the differential Eq. (1) with the moment of $M=F_{\mathrm{g}} z$. As a superposition, for the bending of the pillar it results:

$$
\delta=\frac{F_{\mathrm{L}} h^{3}}{3 E I}+\frac{F_{\mathrm{g}, \mathrm{Cell}} \delta h^{2}}{2 E I}+\frac{F_{\mathrm{g}, \mathrm{Pillar}} \delta h^{2}}{2 E I}
$$

and the total acting force

$$
F=\frac{3}{2} \frac{2 E I-\left(F_{\mathrm{g}, \text { Cell }}+F_{\mathrm{g}, \text { Pillar }}\right) h^{2}}{h^{3}} \delta_{\text {total }}
$$

The gravitational forces are calculated by

$$
\begin{aligned}
F_{\mathrm{g}, \text { Cell }} & =\gamma m_{\text {partial-Cell }} g \\
F_{\mathrm{g}, \text { Pillar }} & =\gamma m_{\text {Pillar }} g .
\end{aligned}
$$

Here, $\gamma$ is the gravitational level, $g$ the gravitational acceleration, $m_{\text {Pillar }}$ the mass of one pillar and $m_{\text {partial-Cell }}$ the partial mass of the cell that acts on one pillar. This partial mass can be calculated by using the ratio of the volumes:

$$
\frac{V_{\text {partial-Cell }}}{V_{\text {Cell }}}=\frac{A_{\text {partial-Cell }} h_{\text {Cell }}}{\frac{\pi}{4} d_{\text {Cell }}^{2} h_{\text {Cell }}}=\frac{m_{\text {partial-Cell }}}{m_{\text {Cell }}} .
$$

The height of the cell, $h_{\text {Cell }}$, with a cylindrical volume cancels out. Thus, the partial mass of the cell only depends on three cell values: the mass, $m_{\text {Cell }}$, the diameter, $d_{\text {Cell }}$, and the partial area, $A_{\text {partial-Cell }}$. The partial area is the part of the cell that acts on one pillar and can be calculated with the geometry of a hexagonal structure (Fig. 4B):

$$
A_{\text {partial-Cell }}=\frac{\sqrt{3}}{2}\left(S+d_{\text {Pillar }}\right)^{2} .
$$

Here, $S$ is the spacing between two pillars and $d_{\text {Pillar }}$ the diameter of the pillar.

The substitution of (9) into (8) yields the mass of the partial cell in (6):

$$
m_{\text {partial-Cell }}=\frac{2 \sqrt{3}}{\pi}\left(\frac{S+d_{\text {Pillar }}}{d_{\text {Cell }}}\right)^{2} m_{\text {Cell }}
$$

The mass of the pillar in (7) is given by

$$
m_{\text {Pillar }}=\rho_{\text {Pillar }} V_{\text {Pillar }} \text {. }
$$

Here, $\rho_{\text {Pillar }}$ is the density of the pillar material and $V_{\text {Pillar }}$ its cylindrical volume. 
bioRxiv preprint doi: https://doi.org/10.1101/2020.03.30.015958; this version posted March 31, 2020. The copyright holder for this preprint (which was not certified by peer review) is the author/funder. All rights reserved. No reuse allowed without permission.

Biophysical Journal Template
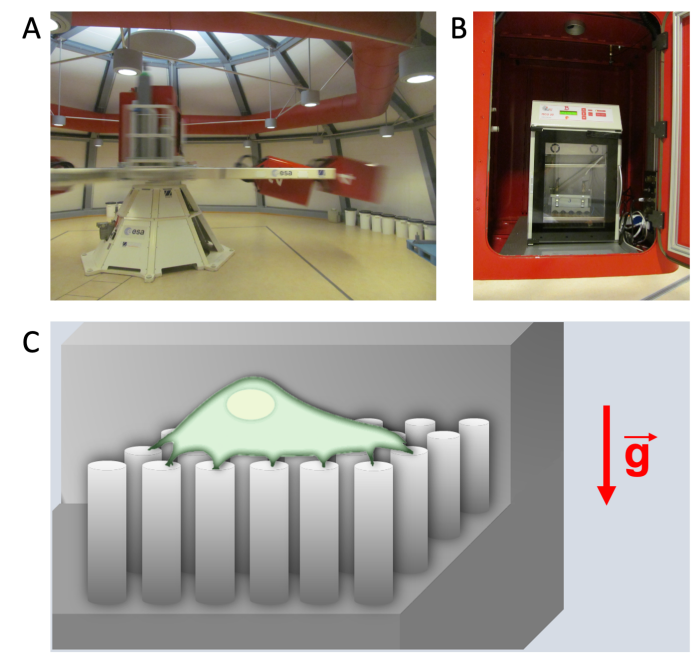

Figure 1: A: Large Diameter Centrifuge (LDC) at ESA/ESTEC provided hypergravity conditions. B: Samples were located in a metal box flushed with $5 \% \mathrm{CO}_{2}$ inside the incubator of on one of the LDC-gondolas. The temperatur was set to $37{ }^{\circ} \mathrm{C}$. $\mathrm{C}$ : Schematic illustration of a cell assembled on top of a micropillar array in upright sample orientation. The g-force was exerted perpendicular to the cell spreading area. 
Eckert et al.
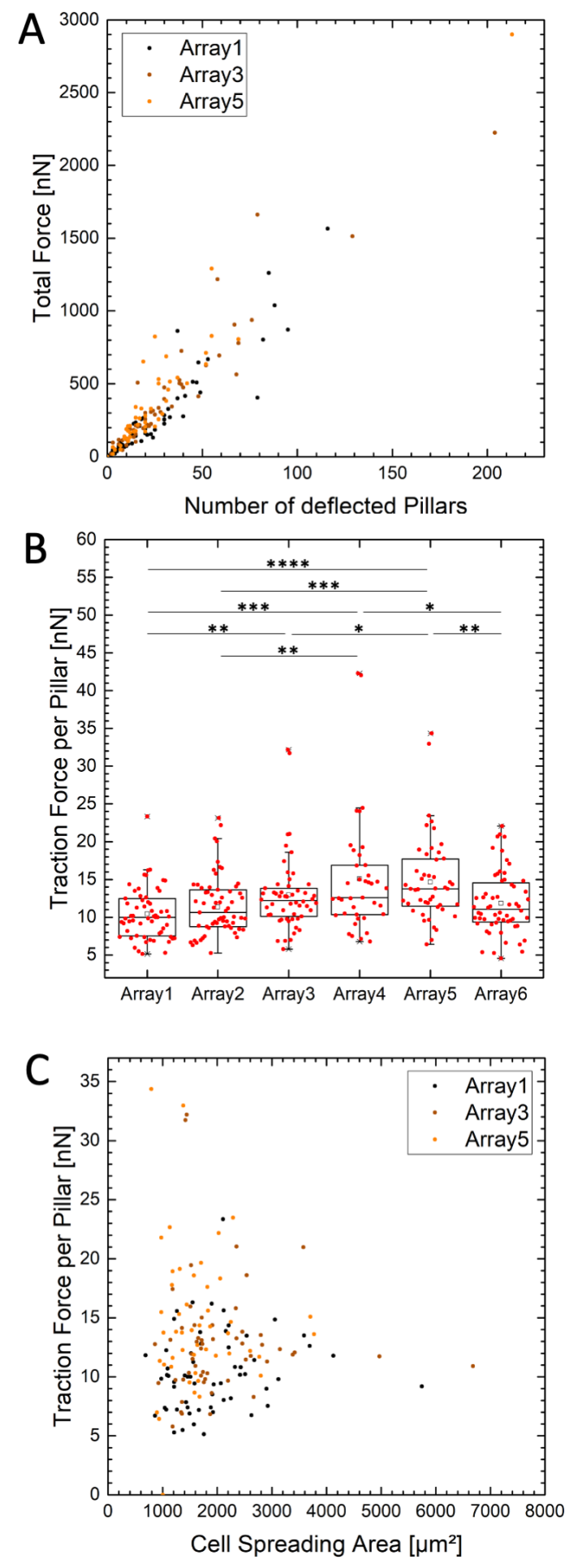

Figure 2: 3 T3 fibroblasts on six independently produced array under standard gravity conditions were analysed. The total force per cell increases linearly with the number of deflected pillars (A). The ratio results in the traction force per deflected pillar (B). This value differes significantly for cells on equally prepared arrays. The traction force is independent from the cell spreading area (C). Three of six datasets randomly chosen are shown in A and C. Each datapoint represent one analysed cell. 

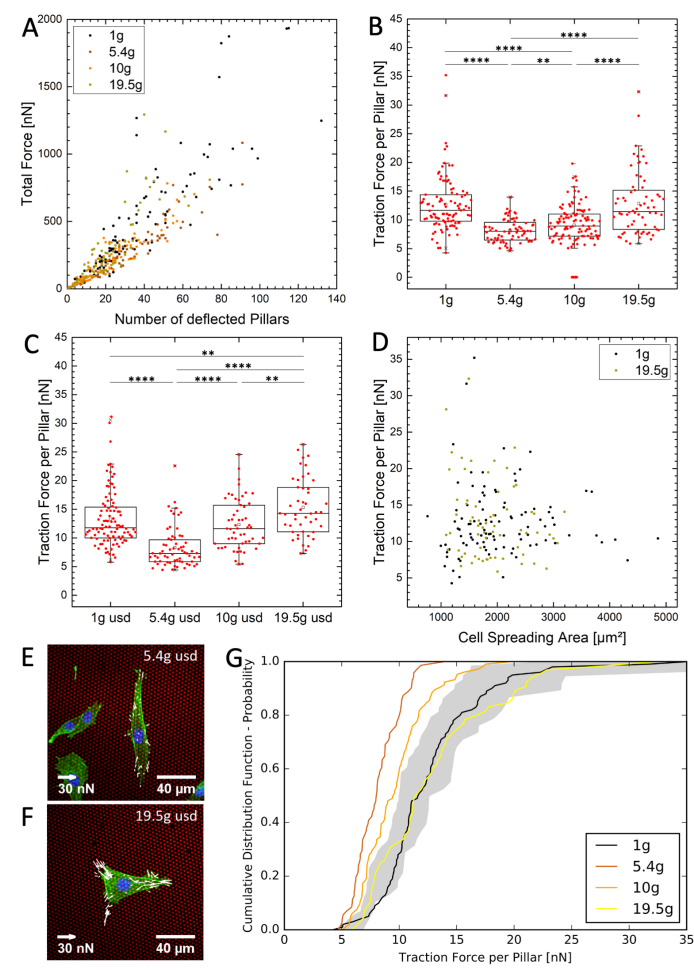

Figure 3: 3T3 firoblasts were exposed to hypergravity in upright and up-side-down orientation. The total force per cell increases linearly with the number of deflected pillars of cells exposed to $1 g, 5.4 g, 10 g$ and $19.5 g$ in upright orientation (A). The ratio results in the traction force per deflected pillar for cells in upright (B) and up-side-down (usd) orientation (C). The traction force decreases from $1 g$ to $5.4 g$ and increases from $5.4 g$ to $19.5 g$. This effect is independent from the cell spreading area (D). Two of four randomly chosen datasets are shown. Each data point represent one analysed cell. In the usd orientation, the biggest difference is between $5.4 g(\mathrm{E})$ and $19.5 \mathrm{~g}(\mathrm{~F})$. Red: pillar array, green: actin filament, blue: nucleus. Compared to experiments under $1 g$ gravity conditions, cells in upright orientation exert less force on pillars at $5.4 g$ and $10 g(\mathrm{G})$. The gray range is the defined force limit for cell traction forces under $1 g$ conditions. This limit is set by the minimal and maximal averaged cell force of the six micropillars at $1 g$ in Fig. 2 B.
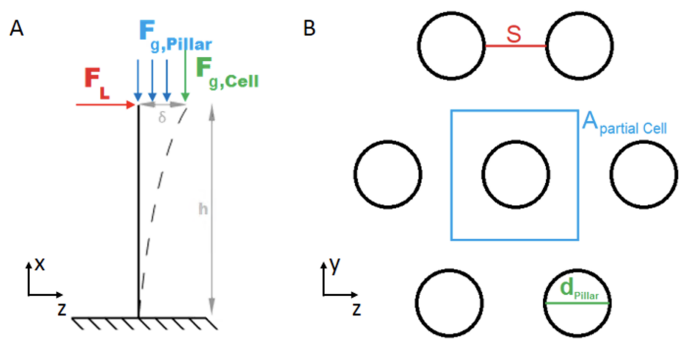

Figure 4: A: Micropillar is described by a cantilevered beam with the height, $h$. A cell sitting on the tip produces a lateral force, $F$, and causes a deflection, $\delta$, of the pillar. In addition, gravitational forces increase the weight of the cell, $F_{\mathrm{g}, \text { Cell }}$, and the pillar, $F_{\mathrm{g} \text {,Pillar }}$, causing a higher deflection. B: Schematic top view of the hexagonal micropillar array. $S$ is the spacing, $d_{\text {Pillar }}$ the diameter of the pillar and $A_{\text {partial-Cell }}$ the partial area of the cell that acts on one single pillar 